\title{
EL USO DEL MODELO FARMACOLÓGICO BASADO EN LA ADMINISTRACIÓN EXPERIMENTAL DE MK-801, PARA LA REVISIÓN Y RE-DEFINICIÓN DEL SISTEMA LÍMBICO EN EL CONTEXTO DE LA ANÁTOMO-PATOLOGÍA
}

\section{THE USE OF THE PHARMACOLOGICAL MODEL BASED ON THE EXPERIMENTAL ADMINISTRATION OF MK-801, FOR THE REVISION AND REDEFINITION OF THE LIMBIC SYSTEM INTO THE ANATOMICAL AND PATHOLOGICAL CONTEXT}

\author{
Adrián Marcelo Bueno \\ Universidad Nacional de Córdoba, Argentina \\ Universidad Católica de Córdoba, Argentina \\ Inst. de Inv. Médica Mercedes y Martín Ferreyra, INIMEC-CONICET,Córdoba, Argentina
}

\author{
Soledad de Olmos \\ Inst. de Inv. Médica Mercedes y Martín Ferreyra, INIMEC-CONICET Córdoba, Argentina
}

\begin{abstract}
Resumen: El efecto neurotóxico inducido en el sistema límbico de ratas por el tratamiento agudo con MK-801, afecta a todas las áreas del lóbulo límbico, como la corteza olfatoria, la amígdala baso-lateral, el sistema hipocampal, la corteza del cíngulo y regiones específicas de la corteza frontal e insular (allocorticales y meso-corticales). En contraste, los núcleos sub-corticales habitualmente incluidos en el sistema límbico, como la amígdala extendida, el sistema estriado-palidal ventral, el área septal, el tálamo y el hipotálamo, no evidencian muerte neuronal. Los resultados anátomo-patológicos sugieren que las áreas del lóbulo límbico comparten características comunes, y soporta la idea de una unidad funcional que es diferente, dentro del sistema límbico, de otras regiones “límbicas” sub-corticales. Esto apoya la consideración de un lóbulo límbico compuesto estrictamente por áreas allo-corticales y meso-corticales del telencéfalo e implica la necesidad de re-definir el clásico y amplio concepto de "sistema límbico".
\end{abstract}

Palabras clave: MK-801, Muerte neuronal, Sistema Límbico, Modelo de Neuropatología.

\begin{abstract}
The neuro-toxic effect induced in the rat limbic system after acute MK-801-treatment, affects all the limbic lobe areas, such as olfactory cortex, basolateral amygdala, hippocampal system, cingulate cortex and specific frontal and insular cortical region (allo-cortical and meso-cortical areas). In contrast, the sub-cortical nucleus usually included into the limbic system, such as the extended amygdala, the estriato-pallidal-ventral system, septal area, thalamus and hippothalamus, do not evidentiate neural death. Anatomo-pathological results suggest that the limbic lobe areas share common characteristics, and support the idea of a different functional unit, which differs from other sub-cortical "limbic" regions. This supports the idea of a limbic lobe formed only by thelencephalic allo-cortical and meso-cortical areas and implies the need to redefine the classic and broad concept of "limbic system".
\end{abstract}

Keywords: MK-801, Neuronal death, Limbic System, Neuropathologic model.

\section{INTRODUCCIÓN}

El MK-801 o dizocilpina maleato es un psicofármaco que actúa como "antagonista no-competitivo de los receptores NMDA glutamatérgicos" (Kemp, Foster y Wong, 1987).

De hecho, este tipo de fármacos bloquea los canales de calcio ubicados en los receptores NMDA, una vez que estos han sido abiertos por la acción del glutamato en su sitio específico.

Como consecuencia, entra menos calcio a la neurona y disminuye la excitabilidad glutamatérgica. La fenciclidina (PCP), conocida vulgarmente como "polvo de ángel", y la ketamina (KTA), habitualmente usada como anestésico, son drogas de abuso (A.P.A., 1995) que tienen una acción análoga a la del MK-801.

De hecho, el interés por estudiar estos psicofármacos está dado, en parte, por su relación con los procesos de adicción a drogas.

Además, el uso médico-clínico que se hace de ellos (como anestésicos o como anticonvulsivantes), y la manifestación de estados disociativos y psicóticos que induce su consumo en

Correspondencia: Adrían Bueno. Cátedra de Neurobiología, Carrera de Psicología, Universidad Católica de Córdoba, Argentina Correo electrónico:adrianmarcelobueno@yahoo.com.ar . Laboratorio de Psicología, Facultad de Psicología, Universidad Nacional de Córdoba.Instituto de Investigación Médica Mercedes y Martín Ferreyra, INIMEC-CONICET, Córdoba, Argentina. Correoelectrónico: immf@immf.uncor.edu 
sujetos humanos, le ha dado cada vez mayor relevancia en el contexto de la neurología y la psiquiatría (Lahti, Holcomb, Medoff y Tamminga, 1995).

En efecto, los "antagonistas NMDA" están siendo usados para modelar psicopatologías en el ámbito de la psicobiología experimental, y representa, desde hace dos décadas, el modelo experimental de esquizofrenia que goza de mayor consenso (p.e. Carlsson y Carlsson, 1990; Olney, Newcomer y Farber, 1999). En este marco, la evaluación de los efectos del MK-801 o sus análogos en el sistema nervioso de animales experimentales, pone en evidencia que, en general, el impacto farmacológico afecta a las mismas estructuras cerebrales que muestran alteraciones anatómicas y morfológicas en sujetos con esquizofrenia (Ellison, 1995). Y en este sentido, las estructuras del sistema límbico son las regiones encefálicas que resultan de mayor interés morfológico y funcional (p.e. Tamminga, Vogel, Gao, Lahtí y Holcomb, 2000).

Ahora bien, en los estudios experimentales en los que se ha evaluado el efecto tóxico del MK-801, PCP o KTA, los conceptos estructurales relacionados al sistema límbico han sido tratados en términos generales, y en el grueso de ellos se ha acotado el análisis sólo a la corteza retroesplenial del cíngulo posterior, seguramente por ser el área más afectada (p.e. Fix, Ross, Stitzel y Switzer, 1996). En gran cantidad de reportes no se han considerado, en detalle, los límites entre las diversas estructuras, lo que ha llevado a algunas confusiones (por ej. Ellison [1995] en la Fig. 1 reporta lesión en el septum ventral, cuando es en la taenia tecta olfatoria).

Aun dentro de la muy estudiada corteza retroesplenial, los autores no definen sus sub-divisiones intra-estructurales, siendo evidente que ambas responden diferentes al impacto tóxico del MK-801 (ver Bueno, de Olmos, Heimer y de Olmos, 2003a [Fig. 8]). Además, los avances en el marco de la neuroanatomía propiciaron la revisión y modificación de conceptos clásicos, lo cual ha sucedido muy particularmente dentro del sistema límbico, en donde se han evidenciado nuevas sub-estructuras y sub-sistemas (ver p.e. Heimer, Harlan, Alheid, García y de Olmos, 1997).

Consecuentemente, las consideraciones funcionales y comportamentales relacionadas a las alteraciones morfológicas inducidas por "antagonistas NMDA" han sido sobre-simplificadas, limitando el alcance teórico de este modelo. Así mismo, se hacen más groseras aún las ya gruesas comparaciones entre los datos experimentales y lo observado en los casos clínicos que se pretenden modelar (para el caso, de esquizofrenia). Por todo ello, cobra relevancia realizar un análisis de las alteraciones estructurales que aparecen tras el tratamiento con MK-801, siguiendo en detalle a las más recientes parcelaciones neuro-anatómicas, y poniendo el foco de atención dentro del sistema límbico, dada su importancia morfo-funcional en el marco de la anátomo-patología.

\section{OBJETIVOS}

Este trabajo tiene dos objetivos, a saber:

a) Evaluar experimentalmente el efecto tóxico del MK-801 en estructuras del sistema límbico de la rata.

b) Revisar el concepto de sistema límbico a la luz de las más recientes consideraciones, y re-definirlo en términos anátomo-patológicos más precisos.

\section{MATERIALES Y METODOS}

Para la realización del trabajo experimental, se han usado ratas hembras adultas $(68+/-8$ días de edad), de la cepa Sprague Dawley, las cuales fueron inyectadas con una dosis única (i.p.) de MK-801 (10 mg/kg) [N=6] o de solución fisiológica ( $\mathrm{ClNa}, 2 \mathrm{ml} / \mathrm{kg}$ ) [N=6]. Todas las ratas fueron sacrificadas 3 días después de la inyección por perfusión trans-cardiaca. Todos estos parámetros fueron ajustados en función de experiencias previas (p.e. Wozniak et al. 1998). El material histológico fue procesado siguiendo estrictamente el protocolo de la técnica Amino-Cupro-Argéntica (A-Cu-Ag), procedimiento aplicado para teñir, de negro, neuronas que evidencian un estado degenerativo como consecuencia de una injuria (de Olmos, Beltramino y de Olmos, 1994). Variados microscopios binoculares fueron el soporte para la evaluación histológica del tejido. Las ilustraciones del material se obtuvieron con técnicas digitales, capturando la imagen con una cámara (Leaf Lumina) montada sobre un microscopio de luz (Zeizz Axioskop), y utilizando como soporte un programa específico de análisis de imagen (Adobe Photoshop).

\section{PARCELACION ANATÓMICA Y NOMENCLATURA DEL SISTEMA LIMBICO}

Si bien los vestigios nominales, morfológicos y funcionales del concepto de "Sistema Límbico" se pueden encontrar en la obra de Broca (1878), 
tal denominación fue incluida en la literatura científica por McLean (1952), ampliando el circuito neuroanatómico y funcional que años antes había sido propuesto por Papez (1937) como la base de los procesos emocionales (ver discusión).

Siguiendo este criterio, el sistema límbico definido por McLean integra a una larga lista de estructuras cerebrales que incluyen al sistema olfatorio completo, la amígdala, la formación hipocampal, la corteza del cíngulo, el lóbulo de la ínsula, la corteza frontal orbital y medial, los núcleos del área septal, la región ventral de los ganglios basales, y sub-núcleos específicos del tálamo y del hipotálamo. Más tarde, McLean (1970) lo llamaría "cerebro mamífero" en el contexto de su concepción del cerebro trino.

Como puede notarse, el sistema límbico así concebido es anatómica, morfológica, funcional y filogenéticamente un territorio muy amplio y heterogéneo, lo cual ha suscitado controversias al momento de definir con precisión sus límites (para una revisión ver Lautin, 2002).

De acuerdo con Gloor (1997), queda claro que la definición de sistema límbico depende del o los criterios que priorice cada autor, lo cual es un tema que será discutido oportunamente en este trabajo.

De todas maneras, en este trabajo se parte de la definición de McLean (1952), por ser la más amplia, y también la más clásica. La evaluación histológica de las estructuras límbicas, se realizó siguiendo la parcelación y la nomenclatura de la última edición del clásico "Atlas Estereotáxico de la rata" de Paxinos y Watson (2006). Con esta parcelación se definieron los límites y la denominación de las regiones anatómicas correspondientes al sistema límbico de la rata, todas las cuales corresponden a las estructuras evaluadas en este trabajo.

\section{RESULTADOS}

En todo el manto de la corteza olfatoria, el efecto tóxico delMK-801 afecta específicamente a neuronas piramidales localizadas en la capa III, cuyas terminales nerviosas pueden verse densamente teñidas en la capa lb, como un reguerocontinuoy paraleloalasuperficie(Fig.1).

Profunda a la corteza olfatoria, se observa degeneración neuronal somática y dendrítica circunscripta a la cápsula superficial del complejo baso-lateral amigdalino (Fig. 1).

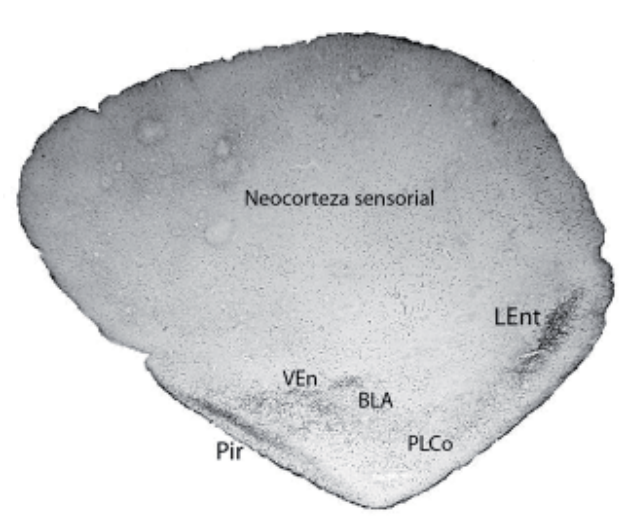

FIGURA 1. Corte para-sagital (lateral 5,80 mm) de un caso experimental, en el que puede observarse degeneración somatodendrítica y terminal en la corteza olfatoria primaria (piriforme [Pir]), en la corteza olfatoria secundaria (división postero-lateral de la amígdala [PLCo]) y en la corteza para-hipocampal (corteza entorhinal lateral [LEnt]). Profundo a la corteza olfatoria, además, se detecta degeneración somato-dendrítica en el núcleo endopeduncular ventral (VEn) y en la cápsula del complejo basolateral amigdalino (BLA). Obsérvese que a esta altura, no se observa degeneración neuronal en la iso-corteza sensorial somática y auditiva. [Aumento $=1,5 \mathrm{x}$ ]

Toda la corteza parahipocampal evidencia, al menos, terminales nerviosas en estado degenerativo en la capa superficial, siendo la corteza entorhinal lateral la subdivisión con mayor densidad de degeneración somato-dendrítca (Fig. 1).

Similar a la corteza olfatoria, se observa tinción de neuronas piramidales en la capa III, aunque en esta región se detectan neuronas estrelladas en la capa II.

Dentro del hipocampo proper se visualiza una densa degeneración de células granulares en el giro dentado (Fig. 2), la cual es concomitante con una tinción de terminales nerviosas que se muestran compactas en diversas capas, tanto del giro dentado como del cuerno de Ammon (ver también Fig. 5). Pueden observarse, además, células piramidales localizadas en el área CA1 y en el complejo subicular (ver Figs. 3 y 5 ).

La corteza retroesplenial del cíngulo posterior (RSC) es, sin duda, la región encefálica que evidencia mayor densidad de muerte neuronal después del tratamiento con MK-801 (Fig. 3).

Gran cantidad de somas de neuronas piramidales aparecen teñidos en las capas intermedias y semi-profundas sobre un denso trasfondo de dendritas, terminales y axones en estado degenerativo. La capa superficial de la RSC evidencia una muy densa degeneración terminal. 
Si bien se observa este patrón degenerativo como un continuo en toda la corteza del cíngulo, la densidad disminuye progresivamente hacia delante, de modo que el cíngulo anterior muestra menos degeneración somato-dendrítica mientras más adelante se lo analiza.

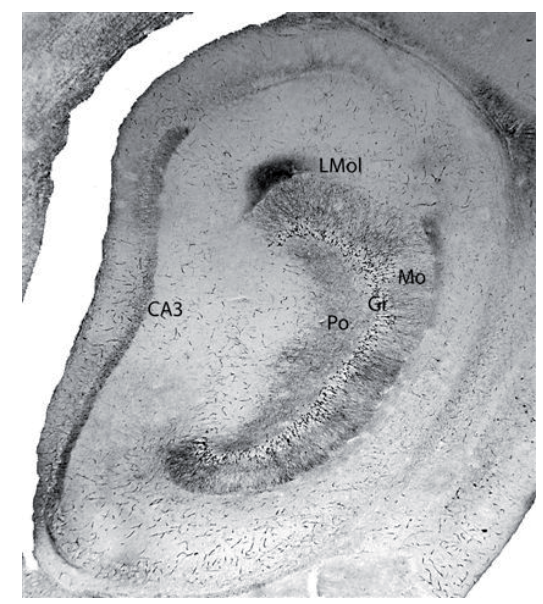

FIGURA 2. Corte para-sagital (lateral $4,60 \mathrm{~mm}$ ) en el que se observa el cuadro degenerativo en el hipocampo proper. El giro dentado muestra cuerpos neuronales en la capa granular (Gr), dendritas en la capa molecular (Mo) y terminales en la capa polimórfica (Po), acompañado de una compacta degeneración terminal en la capa "lacunosa" molecular (LMol). En el cuerno de Ammon también se observan terminales, que en esta foto son visibles sólo en el área CA3 (véanse también las Figs. 3 y 5). [Aumento $=2 \mathrm{x}$ ]

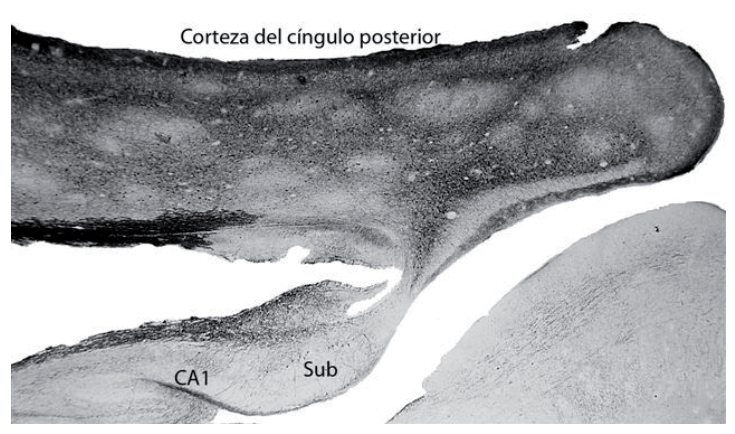

FIGURA 3. Corte para-sagital (lateral $0,80 \mathrm{~mm}$ ) que denota el intenso deterioro de la corteza del cíngulo posterior, consecuente al tratamiento con MK-801. También en esta foto puede verse degeneración somatodendrítica en el complejo subicular (Sub) y en el cuerno de Ammon (CA1). [Aumento = 2 x]

Más aún, al observar la pared medial del lóbulo frontal por adelante del cíngulo anterior, se detectan terminales nerviosas en la capa superficial, pero con escasa o nula degeneración somato-dendrítica (Fig. 4).
El resto de las estructuras encefálicas consideradas parte del sistema límbico, como los núcleos y sub-núcleos de la amígdala extendida, del sistema estriado-palidal ventral, del área septal, del tálamo y del hipotálamo, no evidencian muerte neuronal en ninguno de los casos experimentales (Fig. 5; ver también Fig. 4).

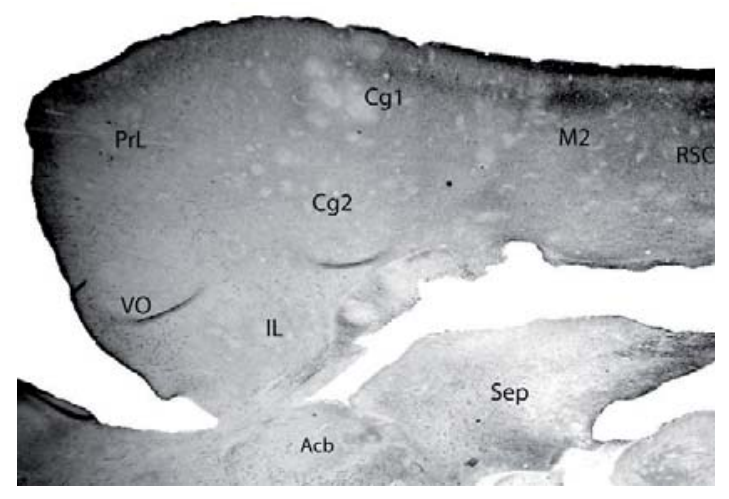

FIGURA 4. Corte para-sagital (lateral 0,70 mm) en el que se observan las regiones anteriores de la pared medial de la meso-corteza. La degeneración somatodendrítica visible en las capas intermedias del cíngulo posterior (RSC) o en la corteza motora secundaria (M2), comienza a disminuir hacia las regiones del cíngulo anterior (Cg1 y Cg2) o la corteza pre-límbica (PrL), hasta casi desaparecer en la corteza orbital ventral (VO) e infra-límbica (IL). Sin embargo, la densidad de terminales nerviosas teñidas en la capa superficial, se mantiene estable en todas las estructuras. [Aumento $=1,5 \mathrm{x}$ ]

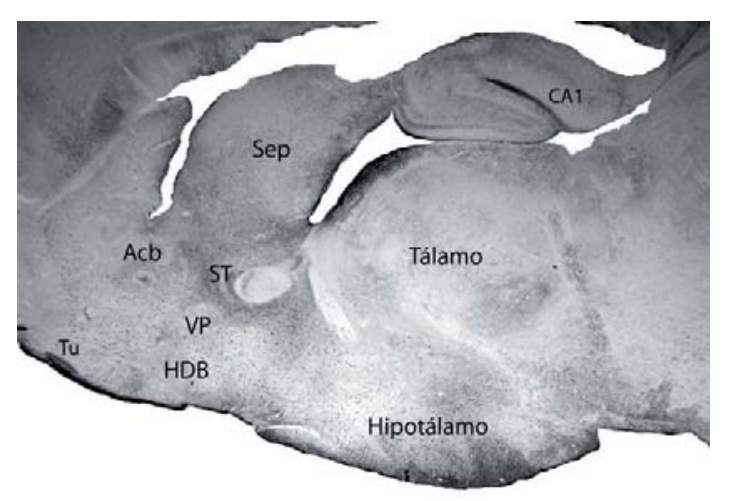

FIGURA 5. Corte para-sagital (lateral 1,30 mm) de un caso experimental, en donde se muestra tinción negativa a la técnica de plata en regiones sub-corticales habitualmente consideradas parte del sistema límbico, como el séptum (Sep), el núcleo accumbens (Acb), la estría terminal de la amígdala extendida (ST), la banda diagonal de Broca (HDB), el pálido ventral (VP), o los diversos sub-núcleos talámicos e hipotalámicos visibles a esta altura. En este corte también se puede ver la degeneración somatodendrítica y terminal detectable en el cuerno de Ammon (CA1) y la degeneración terminal visible en la capa superficial de la corteza olfatoria a la altura del tubérculo (Tu). [Aumento = 1,5 x] 


\section{DISCUSIÓN}

Los datos obtenidos en esta investigación son, en términos generales, consistentes con los reportados en trabajos similares en los que se han usado otras técnicas de evaluación, se han administrado fármacos análogos al MK801 , o se han manipulado combinadamente otras variables, como: edad, sexo, sobrevida, especie, cepa y/o dosis (Olney, Labruyere y Price, 1989; Farber et al. 1995; Fix, et al. 1996; Horváth, Czopf y Buzsáki, 1997; Hur, Son, Shin, Kang y Kim, 1999).

Sin embargo, y como ya se aclarara en la introducción, el análisis anatómico realizado en tales investigaciones fue general y poco preciso, o circunscripto a las áreas de más alto impacto tóxico. El presente trabajo, por el contrario, aporta un análisis con gran detalle en la evaluación neuroanatómica y atendiendo a las más recientes definiciones morfofuncionales. Además, la gran sensibilidad de la técnica $\mathrm{A}-\mathrm{Cu}-\mathrm{Ag}$ para teñir particularmente las terminales nerviosas en estado degenerativo (p.e. Switzer, 2000), ha permitido involucrar en el proceso patológico inducido por MK-801, a áreas específicas de la meso-corteza cuya exclusión hubiera interferido en la interpretación y discusión de los resultados.

Ahora bien, para revisar el concepto de "sistema límbico", es preciso comenzar por la idea original de Paul Broca (1878), quien, trabajando con pequeños roedores, definió como "gran lóbulo límbico" a un grupo de estructuras corticales de la pared medial de los hemisferios cerebrales, dispuestas en forma de anillo alrededor del tálamo y del cuerpo calloso. Dentro de él, el autor incluyó a las cortezas piriforme, amigdalina y para-hipocampal, al hipocampo proper, a la corteza del cíngulo y a las regiones postero-mediales de la corteza frontal. Inicialmente, Broca atribuyó a su lóbulo límbico una función netamente olfatoria, motivo por el que clásicamente se le ha asignado la denominación de "rinencéfalo" o "cerebro olfatorio". A la luz del conocimiento actual, este concepto sería más preciso si se lo limitara sólo a las estructuras antero-ventrales del anillo (Lautin, 2002; Shipley et al. 2004).

Años después, Yakovlev (1948) realizó el que se considera el primer análisis basado en el desarrollo filogenético y en la cito-arquitectura del encéfalo. El autor dividió a la corteza en endopalio, mesopalio y ectopalio.
En el "endopalio" incluyó a estructuras de 3 capas, como la corteza olfatoria y el hipocampo proper, más el "rudimento hipocampal" que conecta a ambas regiones. En el "mesopalio" el autor incluyó a las cortezas para-hipocampal, del cíngulo, frontal medial y orbital, al lóbulo de la ínsula y a las regiones temporales adyacentes. Estas áreas del mesopalio tienen el común denominador de tener un grado de estratificación intermedio entre el endopalio y el ectopalio (en general, de 5 capas). El "ectopalio", en fin, representa la sub-división de más reciente desarrollo, en la que el autor incluyó a todas las áreas corticales con 6 capas celulares bien diferenciadas. La definición de Yakovlev, en este contexto, implica que el endopalio y el mesopalio, juntos, conforman una región homologable al lóbulo límbico de Broca, con una ligera ampliación de sus límites hacia regiones fronto-témporo-insulares.

Estas tres sub-regiones de Yakovlev son respectivamente equivalentes a la allo-corteza, la meso-corteza y la iso-corteza (ver Gloor, 1997; Palomero-Gallagher y Zilles, 2004). Recientes estudios citoarquitectónicos han ajustado aún más los límites entre estas sub-divisiones en áreas específicas, como las cortezas pre-frontal medial, orbito-frontal y témporo-insular, además de incluir al complejo baso-lateral amigdalino (BLA) como un área "pseudo-cortical", diferenciándolo de las estructuras sub-corticales de la amígdala (Heimer y Van Hoesen, 2006; ver también Mega, Cummings, Salloway y Malloy, 1997; de Olmos, Beltramino y Alheid, 2004).

Dada la característica anatómica del lóbulo límbico así definido, en la literatura general se lo denomina también "corteza límbica" (p.e. Vogt, Vogt y Farber, 2004), lo cual lo discrimina claramentedelasestructurassub-corticalesincluidas dentro del concepto clásico de sistema límbico.

Cabe destacar que muchas de las estructuras sub-corticales incluidas por McLean en el sistema límbico también han sido re-conceptualizadas, particularmente en el telencéfalo a partir de la reciente re-definición anatómica de la "sustancia innominada" ("región sin nombre"), como fuera tempranamente denominada esta compleja zona encefálica (Reil, 1809; Reichert, 1859). Y ha sido tal re-definición de la sustancia innominada lo que principalmente hizo surgir nuevos conceptos anátomo-funcionales dentro del sistema límbico (para una revisión ver Heimer et al. 1997).

Uno de ellos, la "amígdala extendida", está compuesta por los clásicos sub-núcleos central 
y medial de la amígdala, y por la extensión que, en forma de arco continuo, incluye a la estría terminal, al núcleo del lecho de la estría terminal, y a sub-regiones específicas de la sustancia innominada (de Olmos et al. 2004; ver también Heimer et al. 1997). Otra concepción es el del "sistema estriado-palidal ventral", formado por los clásicos sub-núcleos del estriado ventral, el núcleo accumbens, el fondus striati y determinados territorios de la sustancia innominada, re-definidos como pálido ventral (PV). Otras estructuras que también fueron incluidas originalmente dentro de la sustancia innominada, como el núcleo basal de Meynert y el núcleo de la banda diagonal de Broca, son actualmente consideradas, junto al Septum propiamente dicho, parte integral del "área septal" (ver también Risold, 2004).

Estas "nuevas" regiones cerebrales distinguidas: la amígdala extendida, el sistema estriado-palidal ventral y el área septal, por la característica morfológica y la disposición espacial, representan núcleos y circuitos sub-corticales, y se los ha clasificado dentro del "telencéfalo basal anterior", al cual se le atribuye la función "efectora" en los comportamientos emocionales (Heimer y van Hoesen, 2006). Si se sigue el criterio de incluir dentro del sistema límbico sólo a estructuras telencefálicas (p.e. Gloor, 1997), las regiones hasta aquí definidas como "lóbulo límbico" y "telencéfalo basal anterior", serían sus dos grandes y únicos componentes.

Sin embargo, el concepto de sistema límbico que aquí se discute es más amplio. Inicialmente, Papez (1937) consideró al sub-núcleo anterior del tálamo y a los cuerpos mamilares del hipotálamo como partes del circuito neuroanatómico propuesto como la base funcional de las emociones. Como se mencionara en la introducción, McLean (1952) partió del "circuito de Papez" incluyendo a las regiones diencefálicas mencionadas dentro de su clásico concepto de sistema límbico. Fue Yakovlev, con sus estudios conexionales, quien reforzara la inclusión de las estructuras talámicas en el sistema límbico, definiendo un "tálamo límbico" con el que amplió, incluso, los límites establecidos por Papez más allá del tálamo anterior (Yakovlev, Locke y Angevine, 1966; para una revisión ver Bentivoglio, Kultas-Ilinsky e Ilinsky, 1993). Por su lado, dado el rol central del hipotálamo en los aspectos "autónomos" y "glandulares" de los procesos emocionales, pasó a ser el eje central del sistema, al punto que en la literatura general todo el hipotálamo es hoy considerado el "núcleo de salida" del sistema límbico (p.e. Guyton, 1999). Así, al tálamo límbico de Yakovlev y al hipotálamo podría denominárselos "diencéfalo límbico".

Por otro lado, siguiendo los patrones de conexión del hipotálamo, también se incluyeron dentro del sistema límbico a estructuras nerviosas del cerebro medio a las que se denominó "mesencéfalo límbico" (Nauta, 1958). Estudios posteriores han llevado a incluir también a los diversos núcleos del sistema catecolaminérgico que proyectan a las estructuras límbicas, por lo cual se ha sugerido el nombre de "sistema límbico extendido" para re-definir a estas regiones del tronco encefálico (Lautin, 2002).

Resulta claro que el criterio funcional de considerar "sistema límbico" a aquellas estructuras relacionadas con los procesos emocionales, ha expandido los límites del mismo hasta regiones muy lejanas a las que considerara inicialmente Papez. Se ha llegado a proponer (¿irónicamente, tal vez?) que todo el sistema nervioso está relacionado de alguna manera con funciones emocionales, por lo que el concepto de sistema límbico resulta obsoleto y, por lo tanto, prescindible (Brodal, 1969). Ciertamente, la definición clásica de sistema límbico resulta, al menos, confusa, pues muchas regiones con función "emocional" también cumplen otro/s rol/es (motriz, sensorial y/o autónomo).

Asímismo,comoseintrodujeraoportunamente, el sistema límbico de Mclean es muy amplio anatómica, funcional y filogenéticamente como para considerarlo una "unidad" en sí misma.

Si se revisan los resultados de este trabajo siguiendo las anteriores definiciones, es claro que el tratamiento experimental con MK-801 induce muerte neuronal sólo en estructuras que forman parte del "lóbulo límbico", pero NO en los núcleos sub-corticales del "telencéfalo basal anterior" o del "diencéfalo límbico". Por ejemplo, en la amígdala, la muerte neuronal es evidente en la sub-división olfatoria y en el complejo baso-lateral, los cuales han sido incluidos dentro del "lóbulo límbico"; sin embargo, la amígdala extendida, considerada parte del "telencéfalo basal anterior", es resistente al impacto tóxico del psicofármaco.

Es también llamativo el hecho de que la toxicidad del MK-801 afecta, sin excepción, a todas las áreas del lóbulo límbico. Todas las sub-divisiones de la allo-corteza y de la meso- 
corteza muestran alguna forma de proceso neuro-degenerativo, lo cual refuerza la idea de que el lóbulo límbico responde como una unidad claramente diferente de otras regiones cerebrales (Broca, 1878; Yakovlev, 1948; Mega et al. 1997; Heimer y Van Hoesen, 2006).

Finalmente, se hace notar que algunos autores sugieren la existencia de dos sub-divisiones "para-límbicas" dentro de este lóbulo límbico, cada una de las cuales con características citoarquitectónicas, filogenéticas y clínicas que le son particulares (Mega et al. 1997). Dado que una de estas sub-divisiones no cumple funciones "emocionales", se ha propuesto que las áreas relacionadas, como la RSC del cíngulo posterior, sean excluidas del lóbulo límbico (Vogt et al. 2004). En este contexto, la evaluación neuro-patológica realizada en este trabajo no es suficiente para aportar conclusiones, aunque la diferencia expresada particularmente dentro de la RSC del cíngulo posterior (RSG vs. RSD), parece un indicio de relevancia y un motivo para desarrollar futuras investigaciones tendientes a dilucidar esta discusión.

\section{CONCLUSION}

Los resultados del presente trabajo muestran que, después del tratamiento experimental con MK-801, las áreas corticales que conforman el lóbulo límbico se ven afectadas de un modo específico y diferencial, si se las compara con otras estructuras "límbicas" sub-corticales, como las del telencéfalo basal anterior o las del diencéfalo, las cuales parecen resistentes al impacto tóxico.

Esto sugiere fuertemente que el lóbulo límbico es una unidad anátomo-patológica particular y diferente dentro del sistema nervioso en general, y sustenta la idea de re-definir al clásico y amplio concepto de "sistema límbico", a los fines de esclarecer más específicamente el rol relacionado a sus estructuras en el contexto de la neuro-patología.

\section{REFERENCIAS}

A.P.A. (1995). Trastornos relacionados con sustancias. Manual diagnóstico y estadístico de los trastornos mentales. Ed. Masson, 181-278.

Bentivoglio, M., Kultas-llinsky, K., \& llinsky, I. (1993). Limbic thalamus: Structure, intrinsic organization, and connections. Neurobiology of cingulate cortex and limbic thalamus. Ed. Vogt and Gabriel. Cap. 2, 71-122.
Broca, P. (1878). Anatomie comparée des circonvolutions cérébrales. Le grand lobe limbique et la scissure limbique dans la série des mammiféres. Rev Anthrop, 21, 384-498.

Brodal, A. (1969). Neurological anatomy in relation to clinical medicine. Ed. Oxford University Press.

Carlsson, M., \& Carlsson, A. (1990). Schizophrenia: A subcortical imbalance syndrome? Schizophrenia Bull, 18, 425-532.

de Olmos, J., Beltramino, C., \& de Olmos, S. (1994). Use of Amino-Cupric-Silver technique for the detection of early and semiacute neuronal degeneration caused by neurotoxicants, hypoxia, and phisical trauma. Neurotoxicol Teratol, 16, 545-561.

de Olmos, J. Beltramino, C., \& Alheid, G. (2004). Amygdala and Extended Amygdala of the Rat: A Cytoarchitectonical, Fibroarchitectonical, and Chemoarchitectonical Survey. The rat nervous system. Ed. Elsevier. Cap. 19, 509-603.

Ellison, G. (1995). The NMDA antagonist phenciclidine, ketamine and dizocilpine as both behavioral and anatomical models of the dementias. Brain Res. Rev, 20, 250-267.

Farber, N., Wozniak, D., Price, M., Labruyere, J., Huss, J., Peter, H., \& Olney, J. (1995). Age-specific neurotoxicity in the rat associated with NMDA receptor blockade: potential relevance to schizophrenia? Biol Psychiatry, 38, 788-796.

Fix, A., Ross, J., Stitzel, S., \& Switzer, R. (1996). Integrated evaluation of central nervous system lesions: stains for neurons, astrocytes, and microglia reveal the spatial and temporal features of MK801-induced neuronal necrosis in the rat cerebral cortex. Toxicol Pathol, 24, 291-304.

Gloor, P. (1997). The temporal lobe and limbic system. Ed. Oxford.

Guyton, A. (1999). Anatomía y fisiología del sistema nervioso. Ed. Médica Panamericana.

Heimer, L., Harlan, R., Alheid, G., García, M., \& de Olmos, J. (1997). Substantia innominada: A notion which impedes clinical-anatomical correlations in neuropsychiatric disorders. Neurosci, 76, 957-1006.

Heimer, L., \& van Hoesen, G. (2006). The limbic lobe and its output channels: implications for emocional functions and adaptativebehavior. NeurosciBiobehavRev, 30,126-147.

Horváth, Z., Czopf, J., \& Buzsáki, G. (1997). MK801-induced neuronal damage in rats. Brain Res, 753:181-195.

Hur, G., Son, W., Shin, S., Kang, J., \& Kim, Y. (1999). Sex differences in dizocilpine (MK801) neurotoxicity in rats. Environm Toxicol Pharmacol, 7, 143-146.

Kemp, J., Foster, A., \& Wong, E. (1987). Non-competitive antagonists of excitatory aminoacid receptors. Trends Neurosci, 10, 294-298.

Lahti, A., Holcomb, H., Medoff, C., \& Tamminga, C. (1995). Ketamine activates psychosis in schizophrenia and alters blood flow in human limbic cortex. NeuroReport, 6 , 869-872.

Lautin, A. (2002). The limbic brain. Ed. Kluwer Academic Publishers. 
McLean, P. (1952). Some psychiatric implications of physiological studies on the frontotemporal portion of the limbic system (visceral brain). Electroencephalogr Clin Neurophysiol, 4, 407-418.

McLean, P. (1970). The triune brain, emotion and scientific bias, in the neurosciences: second study program. Ed. F. Schmitt. 336-349.

Mega, M., Cummings, J., Salloway, S., \& Malloy, P. (1997). The limbic system: an anatomic, phylogenetic, and clinical perspective. J Neuropsychiat Clin Neurosci, 9, 315330.

Nauta, W. (1958). Hipocampal projections and related neural pathways to the mid-brain in the cat. Brain, 81, 319-340.

Olney, J., Labruyere, J., \& Price, M. (1989). Pathological changens induced in cerebrocortical neurons by phenciclidine and related drugs. Science, 244, 1360-1362.

Olney, J., Newcomer, J., \& Farber, N. (1999). NMDA receptor hypofunction model of schizophrenia. J Psychiatr Res, 33, 523-533.

Palomero-Gallagher, N., \& Zilles, K. (2004) Isocortex. The rat nervous system. Ed. Elsevier. Cap. 23, 729-757.

Papez, J. (1937). A proposed mechanism of emotion. Arch Neurol Psychiatry, 38, 725-733.
Paxinos, G., \& Watson, C. (2006). The rat brain in stereotaxic coordinates. Academic Press.

Reichert, K. (1859). Der Baus des Menschlichen Gehirns durch Abbildung mit erlauterndem. Texte 2. Ed. Engelmann, Leipzig.

Reil, J. (1809). Untersuchungen über den Bau des grossen Gehirn im Menschen. Arch Physiol, 9, 136-208.

Risold, P. (2004). The Septal Region. The rat nervous system. Ed. Elsevier. Cap. 20, 605-632.

Switzer III, R. (2000), Application of silver degeneration stains for neurotoxicity testing. Toxicol Pathol, 28, 70-83.

Tamminga, C., Vogel, M., Gao, X., Lahti, A., \& Holcomb, H. (2000) The limbic cortex in schizophrenia: focus on the anterior cingulate. Brain Res Rev, 31, 364-370.

Vogt, B., Vogt, L., \& Farber, N. (2004). Cingulate cortex and disease models. The rat nervous system. Ed. Elsevier. Cap. 22, 705-726.

Yakovlev, P. (1948). Motility, behavior, and the brain. J Nerv ment Dis, 107, 313-335.

Yakovlev, P., Locke, S., \& Angevine, J. (1966). The limbus of the cerebral hemisphere, limbic nuclei of the thalamus, and the cingulum bundle. The Thalamus. Ed. Columbia University Press. 77-97. 